\title{
Diagnóstico citogenético de pacientes com retardo mental idiopático
}

\author{
Cytogenetic diagnosis of patients with idiopathic mental retardation
}

Natália Duarte Linhares'; Marta Svartman²; Eugênia Ribeiro Valadares ${ }^{3}$

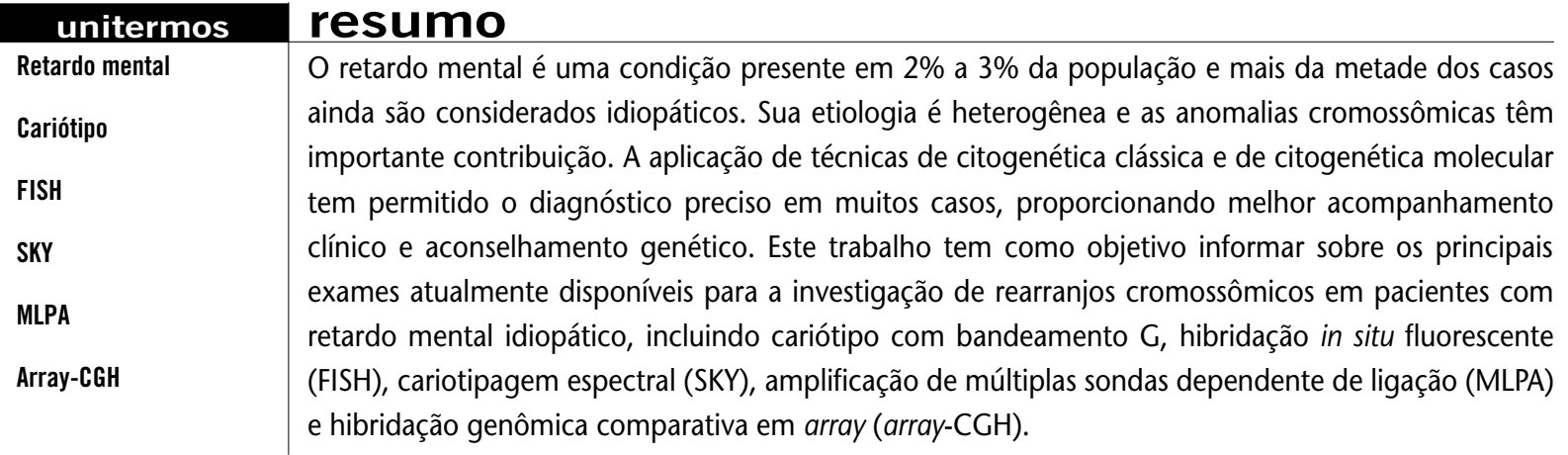

abstract

Mental retardation is a condition that affects $2 \%-3 \%$ of the population and more than half of the cases are still deemed idiopathic. Its etiology is heterogeneous and chromosome abnormalities play a significant role. The application of classical cytogenetic and molecular cytogenetic techniques has enabled accurate diagnosis in several cases, which allows better clinical monitoring and genetic counseling. This paper aims at informing about the major tests currently available to investigate chromosome abnormalities in patients with idiopathic mental retardation, including GTG-banded karyotyping, fluorescence in situ hybridization (FISH), spectral karyotyping (SKY), multiplex ligation-dependent probe amplification (MLPA) and array-comparative genomic hybridization (array-CGH).
Primeira submissão em 04/06/11 Última submissão em 18/08/11 Aceito para publicação em 24/08/11 Publicado em 20/02/12 


\section{Introdução}

O retardo mental (RM), definido pela Organização Mundial da Saúde (OMS) por um quociente de inteligência (QI) menor que 70, é caracterizado por comprometimento de habilidades manifestadas durante $\mathrm{O}$ período de desenvolvimento e que contribuem para o nível global de inteligência, ou seja, habilidades cognitivas, de linguagem, motora e sociais (Classificação Internacional de Doenças, CID-10, versão 2007). O RM manifesta-se antes dos 18 anos e não pode ser diagnosticado antes de a criança completar 5 anos, quando as medidas de inteligência são confiáveis para se aplicar o teste de QI. Para crianças com menos de 5 anos, o termo atraso de desenvolvimento neuropsicomotor (ADNPM) é usualmente utilizado ${ }^{(36)}$.

O RM é uma condição presente em $2 \%$ a $3 \%$ da população geral ${ }^{(31)}$. Anomalias cromossômicas são detectadas em $4 \%$ a $28 \%$ dos casos, dependendo da seleção dos pacientes e da sensibilidade das técnicas empregadas $(5,7,50)$. A síndrome do cromossomo $X$ frágil, causada pela expansão da repetição CGG no gene FMR1, é causa frequente de RM especialmente em homens, com prevalência de $1 / 4.000$ homens $^{(48)}$. O exame citogenético para deteç̧ão do sítio frágil em Xq27.3 não é mais recomendado por apresentar baixa sensibilidade e especificidade ${ }^{(1)}$. Nesse caso, o diagnóstico é estabelecido por exame molecular ${ }^{(9,27)}$.

Mais de $50 \%$ dos casos de RM ainda são considerados idiopáticos ${ }^{(8)}$. Entretanto, o uso de metodologias mais recentes evidenciaram que $10 \%$ a $25 \%$ dos casos de RM envolvem rearranjos muito pequenos, subteloméricos ou intersticiais ${ }^{(25)}$. Rearranjos crípticos envolvendo as regiões subteloméricas são provavelmente as causas mais frequentes de RM idiopático, detectados em aproximadamente 5\% de um total de 2.672 indivíduos analisados ${ }^{(8,26)}$.

As consequências clínicas de um rearranjo cromossômico estão geralmente relacionadas com a localização desse rearranjo, seu tamanho e a quantidade de genes envolvidos e sua função. A alta incidência de deleções subteloméricas reflete o fato de que, devido à riqueza de genes nessas regiões, uma microdeleção subtelomérica tem maior probabilidade de causar um efeito fenotípico, mais comumente o $\mathrm{RM}^{(32)}$. Além disso, são áreas particularmente propensas à instabilidade genômica devido às sequências repetitivas nelas encontradas ${ }^{(24,30)}$.

\section{Métodos de investigação de rearranjos cromossômicos}

O cariótipo com bandeamento $G$ é o exame citogenético inicial indicado para pacientes com suspeita de rearranjos cromossômicos ${ }^{(36)}$. Os cromossomos metafásicos dos pacientes são analisados com resolução de 400 a 550 bandas. No entanto, alterações cromossômicas que afetam segmentos menores que $5 \mathrm{Mb}$, denominadas crípticas, não são detectadas nesse nível de resolução. Além disso, alterações maiores podem passar despercebidas dependendo do padrão de bandas das regiões afetadas. Para melhorar a resolução das metáfases analisadas, Yunis desenvolveu uma técnica para o estudo dos cromossomos humanos em alta resolução, com 650 a 850 bandas, aumentando a possibilidade de detecção de anormalidades menores ${ }^{(51)}$. Porém, esse é um método demorado, que exige muita experiência do citogeneticista e que, por isso, não é apropriado para a análise de rotina.

O advento da técnica de hibridação in situ fluorescente (FISH) aumentou a resolução da análise cromossômica, permitindo a detecção de regiões específicas, e desse modo complementou o exame de citogenética clássica ${ }^{(46)}$. Nas análises de FISH, sondas de DNA de sequências homólogas às sequências-alvo são marcadas com fluorocromos $\mathrm{e}$ hibridadas em cromossomos metafásicos ou em células interfásicas do paciente para determinar presença, localização e número das sequências genômicas analisadas ${ }^{(45,47)}$. Essa técnica tem sido amplamente utilizada para identificar anomalias cromossômicas submicroscópicas, como nas síndromes de microdeleção; entre estas podem ser citadas as síndromes de Prader-Willi, Angelman, velocardiofacial, DiGeorge e Williams ${ }^{(37)}$. Nesses casos, o exame de FISH requer precisão na suspeita clínica, já que são analisadas somente as sequências de interesse.

Utilizando-se sondas de segmentos clonados, como, por exemplo, em cromossomos artificiais de bactéria (BAC) cobrindo a extensão da região cromossômica alterada pela técnica de FISH, pode-se obter uma delimitação muito mais precisa da sequência alterada e de seus pontos de quebra $^{(2,10,22)}$. A identificação exata da região cromossômica duplicada ou deletada é importante para a correlação genótipo-fenótipo.

Com o objetivo de "pintar" simultaneamente cada cromossomo de uma cor, foram desenvolvidas as técnicas de cariotipagem espectral (SKY) ${ }^{(34)}$ e multiplex (M)-FISH ${ }^{(44)}$. Essas metodologias utilizam sondas de "pintura cromossômica" 
de cada um dos cromossomos humanos marcadas por combinações de fluorocromos distintos. As 24 sondas são reunidas em um coquetel, pré-hibridadas com um DNA rico em sequências repetitivas (p. ex, Cot-1 DNA) para evitar hibridações cruzadas, e então são hibridadas a metáfases do paciente. As imagens são capturadas em um microscópio de fluorescência, passando por um interferômetro na técnica de SKY ou utilizando uma série de filtros de excitação e emissão na técnica de M-FISH. Os dados são processados com um software específico, que gera uma imagem em que cada cromossomo é marcado com uma cor diferente. A limitação dessas técnicas está na incapacidade de detectar rearranjos intracromossômicos, como deleções e inversões. Além disso, a faixa de detecção é limitada pela resolução do cariótipo do paciente sendo analisado e os centrômeros e braços curtos dos cromossomos acrocêntricos não sofrem hibridação devido à supressão de sequências repetitivas. Essas técnicas têm sido utilizadas para esclarecer alterações citogenéticas detectadas previamente no cariótipo com bandeamento $\mathrm{G}$, incluindo cromossomos marcadores, translocações e rearranjos complexos ${ }^{(35)}$.

A técnica de amplificação de múltiplas sondas dependente de ligação (MLPA) permite a deteç̧ão do número de cópias de até 40 sequências de DNA genômico em uma única reação baseada na reação de polimerização em cadeia $(\mathrm{PCR})^{(33)}$. Cada sequência-alvo é identificada por meio de um par de sondas que hibridam com o DNA do paciente em regiões adjacentes. Após a adição de ligase, que faz a junção das duas sondas, e posterior desnaturação, estas são amplificadas por PCR utilizando um primer universal marcado com um fluorocromo. A separação e a quantificação dos produtos de amplificação são feitas por eletroforese capilar. A identificação dos produtos é realizada com base no fato de que cada um deles tem tamanho distinto devido a uma sequência stuffer de tamanho conhecido inserida em cada par de sondas. A MLPA tem a vantagem de ser um método de análise fácil e rápida, podendo também detectar facilmente deleções/duplicações presentes no genoma. Entre suas limitações estão a incapacidade de detectar rearranjos balanceados, a dificuldade de detectar mosaicismos baixos (menos de $40 \%$ das células alteradas) e a sensibilidade da técnica em relação à qualidade do DNA utilizado(21).

A hibridação genômica comparativa (CGH) foi desenvolvida inicialmente como uma ferramenta molecular para identificar perdas e ganhos de segmentos cromossômicos em uma avaliação global do genoma de células tumorais ${ }^{(12)}$. $\mathrm{Na} \mathrm{CGH}$, o DNA genômico total do paciente em estudo, marcado com um fluorocromo verde, juntamente com o
DNA genômico total de um indivíduo normal (controle), marcado com um fluorocromo vermelho, são hibridados em metáfases de um indivíduo com cariótipo normal. A análise é realizada com um programa que gera perfis de razão da fluorescência verde:vermelha ao longo de cada cromossomo, permitindo identificar os locais em que houve ganhos ou perdas de material genético. Desse modo, a análise da CGH em metáfase tem aproximadamente a mesma resolução do exame de cariótipo, e rearranjos cromossômicos desbalanceados com uma resolução de aproximadamente 3-10 Mb podem ser detectados ${ }^{(13)}$. Apesar disso, a CGH é um método de diagnóstico muito útil, solucionando 10\% de casos idiopáticos de anomalias congênitas/atraso no desenvolvimento e também de casos de $\mathrm{RM}^{(6,14)}$.

Na busca de maior resolução para análise do número de cópias de segmentos do genoma, foram desenvolvidos métodos de CGH que usam segmentos genômicos impressos ordenadamente em múltiplas áreas de uma lâmina de vidro é o chamado array- $\mathrm{CGH}^{(28,43)}$. A resolução genômica dos diferentes arrays é determinada pelo espaçamento e pelo tamanho de suas sondas, as quais podem ser sequências de DNA genômico humano inseridas em BAC (tamanho de 80-200 Kb) ou pequenas sequências de DNA sintetizadas, como os oligonucleotídeos (25-85 bp). Por exemplo, em um array de oligonucleotídeo de 60K, são impressos na lâmina 60 mil oligonucleotídeos, cobrindo toda a extensão do genoma. Como o genoma humano apresenta aproximadamente $3,2 \mathrm{~Gb}$, o espaçamento médio entre cada oligonucleotídeo é de aproximadamente $50 \mathrm{~Kb}$.

No array-CGH, quantidades equimolares de DNA genômico da amostra do paciente e do controle são co-hibridadas no array genômico, em vez de serem hibridadas em metáfases. Dessa forma, é possível detectar perdas e ganhos de determinados segmentos cromossômicos, mas não o tipo de rearranjo cromossômico que os originou. Por meio de um tipo especial de array, o polimorfismo de nucleotídeo simples (SNP) array, é possível a identificação de dissomias uniparentais de regiões genômicas, além de alterações de um único nucleotídeo e de segmentos cromossômicos maiores ${ }^{(29,42)}$. Porém, essas técnicas não detectam rearranjos balanceados, como inversões e translocações, alterações centroméricas e alterações de células esporádicas. Uma vez detectado o desequilíbrio cromossômico, é preciso complementar o exame com outros métodos, como análises por FISH, MLPA ou PCR quantitativo, a fim de confirmar o achado e definir sua origem ${ }^{(23)}$.

Portanto, o array-CGH é um método que possibilita uma análise refinada de todo o genoma, sem necessitar 
de cultura de células. Por esse motivo, tem facilitado o diagnóstico e a identificação das bases moleculares de várias alterações genéticas ${ }^{(4,38,41,49)}$. Entretanto, a interpretação dos resultados é difícil, já que muitas das alterações detectadas são copy number variations (CNVs) sem significado patogênico ou de significado clínico desconhecido. As CNVs são variações de número de cópias de segmentos de DNA com tamanho maior que $1 \mathrm{~Kb}$, que ocorrem em cerca de $6 \%$ do genoma humano ${ }^{(39)}$. Para confirmar se a CNV encontrada está relacionada com o fenótipo do paciente são necessárias várias análises: verificar se os pais não apresentam CNV; se a CNV é encontrada em indivíduos com o mesmo fenótipo e não em indivíduos saudáveis; se os genes presentes na região alterada estão relacionados com o fenótipo do paciente ${ }^{(23)}$. Para isso, vários bancos de dados estão disponíveis na internet, como http://www.ensembl.org; http://projects.tcag.ca/variation; http://genome.ucsc.edu; http://www.sanger.ac.uk/PostGenomics/decipher ${ }^{(3)}$.

Com o objetivo de facilitar o diagnóstico de crianças com suspeita de rearranjo cromossômico subtelomérico, é possível a avaliação simultânea de todas as sequências subteloméricas por meio do FISH multiprobe subtelomérico ${ }^{(16,17)}$. Nessa técnica, sondas específicas das regiões subteloméricas dos braços curtos e longos de todos os cromossomos humanos, exceto dos braços curtos dos cromossomos acrocêntricos, são multi-hibridadas em células do paciente em metáfase ou intérfase. Com o intuito de realizar uma triagem de todas as regiões subteloméricas em uma única reação/experimento, outras técnicas similares surgiram, como o MLPA subtelomérico e o array-CGH subtelomérico. Essas técnicas são amplamente utilizadas no diagnóstico de pacientes de RM idiopático ${ }^{(15,19,20,40)}$.

Knight et al. utilizaram a técnica de FISH com sondas subteloméricas para examinar a integridade dessas regiões em 284 crianças com RM idiopático moderado a grave, com QI menor que 50, e em 182 crianças com RM idiopático leve, com QI entre 50 e $70^{(18)}$. Rearranjos cromossômicos subteloméricos foram observados em $7,4 \%$ das crianças com RM moderado a grave e em $0,5 \%$ das crianças com RM leve. As alterações eram herdadas em quase $50 \%$ dos casos. Desse modo, foi demonstrado que as causas do RM variam com sua gravidade: pacientes com RM moderado a grave são geralmente relacionados com uma única causa patológica, como rearranjo subtelomérico; já pacientes com RM leve geralmente têm etiologia multifatorial.

\section{Conclusão}

Exames com base em array (incluindo o array-CGH e SNP array) possibilitam o diagnóstico de $15 \%$ a $20 \%$ dos indivíduos com RM/ADNPM, distúrbios do espectro do autismo e anomalias congênitas múltiplas ${ }^{(25)}$. Já o cariótipo com bandeamento $G$ detecta alterações em cerca de 3\% dos casos não associados a síndromes cromossômicas reconhecíveis clinicamente, como a síndrome de Down. Dessa forma, atualmente, exames com base em array têm sido propostos como exames primários para pacientes com RM/ADNPM idiopático ${ }^{(11,25) .}$

A utilização de técnicas baseadas no array na pesquisa e no diagnóstico tem levado à identificação de várias síndromes novas, expandido o espectro fenotípico de síndromes já conhecidas, elucidado as bases genômicas de condições clínicas bem estabelecidas e esclarecido os mecanismos moleculares de algumas alterações cromossômicas ${ }^{(3)}$. Entretanto, deve-se ter cautela na interpretação dos resultados de exames por ser frequente a identificação de alterações de significado clínico desconhecido ou sem importância clínica.

Todas as técnicas previamente descritas, embora de difícil acesso, são realizadas em laboratórios de referência e de pesquisa no Brasil, mas necessitam ser mais bem incorporadas pelo Sistema Único de Saúde (SUS). As principais vantagens e desvantagens de cada uma delas estão listadas na Tabela. Cabe ao médico, especialmente ao geneticista clínico, conhecê-las o suficiente para requisitar os exames mais adequados para seu paciente e saber interpretar seus resultados. Também é fundamental que o laboratório ofereça apoio ao médico para a interpretação certa dos resultados. O diagnóstico correto permite que o paciente e seus familiares recebam acompanhamento e aconselhamento genético adequados.

\section{Referências}

1. AMERICAN COLLEGE OF MEDICAL GENETICS. Standards and guidelines for clinical genetics laboratories. Technical standards and guidelines for fragile $X$ testing: a revision to the disease-specific supplements to the standards and guidelines for clinical genetics laboratories of the American College of Medical Genetics, 2006.

2. ANTONINI, S. et al. Delimitation of duplicated segments and identification of their parental origin in two partial 


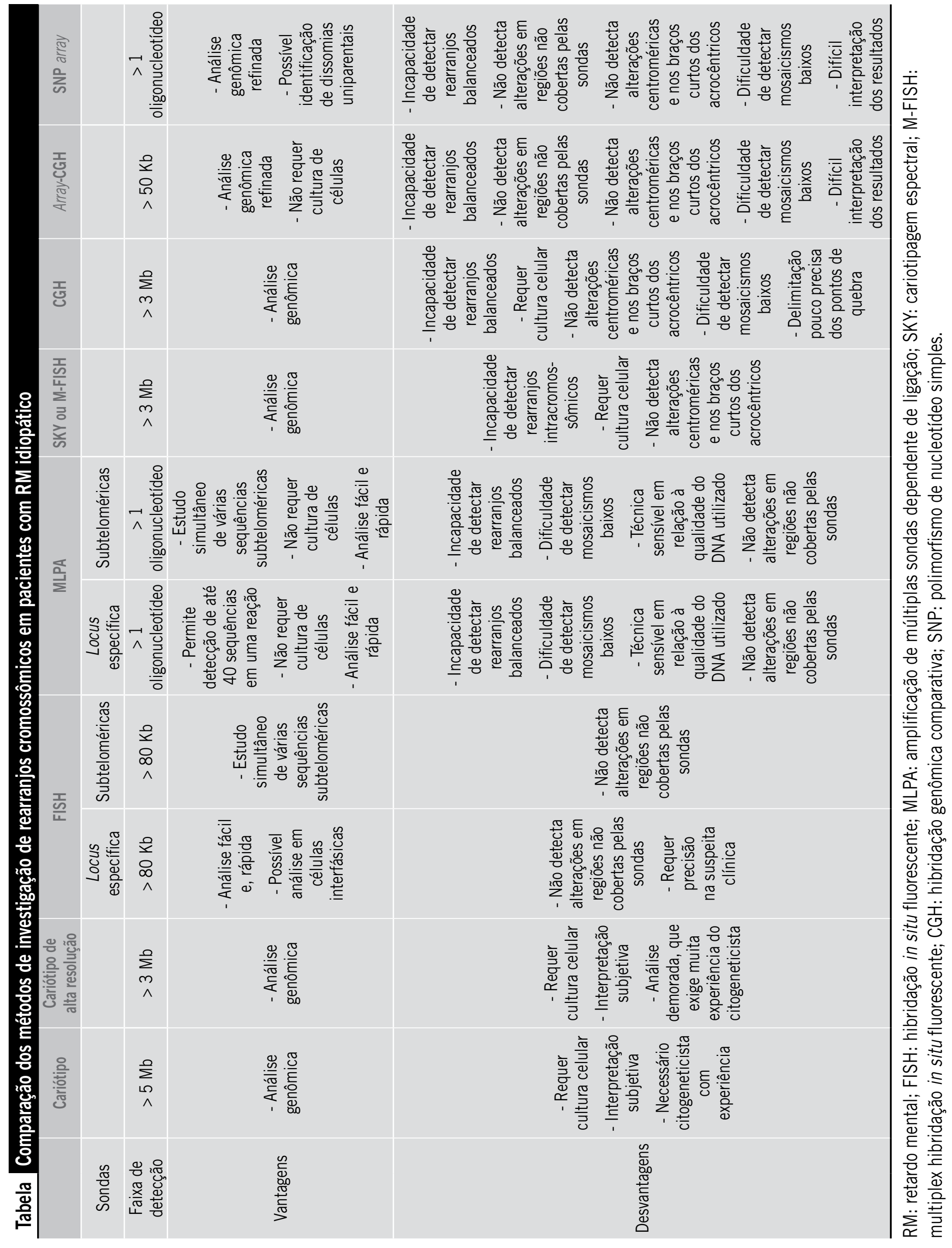


chromosome 3p duplications. Am J Med Genet, v. 113, n. 2, p. 144-50, 2002.

3. BEJJANI, B. A.; SHAFFER, L. G. Clinical utility of contemporary molecular cytogenetics. Annu Rev Genomics Hum Genet, v. 9, p. 71-86, 2008.

4. CHEUNG, S. W. et al. Development and validation of a CGH microarray for clinical cytogenetic diagnosis. Genet Med, v. 7, n. 6, p. 422-32, 2005.

5. CURRY, C. J. et al. Evaluation of mental retardation: recommendations of a Consensus Conference: American College of Medical Genetics. Am J Med Genet, v. 72, n. 4, p. 468-77, 1997.

6. DE VRIES, B. B. et al. Diagnostic genome profiling in mental retardation. Am J Hum Genet, v. 77, n. 4, p. 606-16, 2005.

7. DE VRIES, B. B. et al. Screening and diagnosis for the fragile $X$ syndrome among the mentally retarded: an epidemiological and psychological survey. Collaborative Fragile X Study Group. Am J Hum Genet, v. 61, n. 3, p. 660-7, 1997.

8. DE VRIES, B. B. et al. Telomeres: a diagnosis at the end of the chromosomes. J Med Genet, v. 40, n. 6, p. 38598, 2003.

9. DE VRIES, B. B. et al. The fragile X syndrome. J Med Genet, v. 35, n. 7, p. 579-89, 1998.

10. FLORIDIA, G. et al. The same molecular mechanism at the maternal meiosis I produces mono- and dicentric $8 p$ duplications. Am J Hum Genet, v. 58, n. 4, p. 78596, 1996.

11. HOCHSTENBACH, R. et al. Array analysis and karyotyping: workflow consequences based on a retrospective study of 36,325 patients with idiopathic developmental delay in the Netherlands. Eur J Med Genet, v. 52, n. 4, p. 161-9, 2009.

12. KALLIONIEMI, A. et al. Comparative genomic hybridization for molecular cytogenetic analysis of solid tumors. Science, v. 258, n. 5083, p. 818-21, 1992.

13. KIRCHHOFF, M. et al. Deletions below 10 megabasepairs are detected in comparative genomic hybridization by standard reference intervals. Genes Chromosomes Cancer, v. 25, n. 4, p. 410-3, 1999.

14. KIRCHHOFF, M. et al. High resolution comparative genomic hybridisation in clinical cytogenetics. J Med Genet, v. 38, n. 11, p. 740-4, 2001.

15. KIRCHHOFF, M. et al. Investigation of patients with mental retardation and dysmorphic features using comparative genomic hybridization and subtelomeric multiplex ligation dependent probe amplification. Am J Med Genet A, v. 139, n. 3, p. 231-3, 2005.

16. KNIGHT, S. J. et al. An optimized set of human telomere clones for studying telomere integrity and architecture. Am J Hum Genet, v. 67, n. 2, p. 320-32, 2000.

17. KNIGHT, S. J. et al. Development and clinical application of an innovative fluorescence in situ hybridization technique which detects submicroscopic rearrangements involving telomeres. Eur J Hum Genet, v. 5, n. 1, p. 1-8, 1997.

18. KNIGHT, S. J. et al. Subtle chromosomal rearrangements in children with unexplained mental retardation. Lancet, v. 354, n. 9191, p. 1676-81, 1999.
19. KNIGHT, S. J.; FLINT, J. Perfect endings: a review of subtelomeric probes and their use in clinical diagnosis. J Med Genet, v. 37, n. 6, p. 401-9, 2000.

20. KOOLEN, D. A. et al. Screening for subtelomeric rearrangements in 210 patients with unexplained mental retardation using multiplex ligation dependent probe amplification (MLPA). J Med Genet, v. 41, n. 12, p. 892-9, 2004.

21. KOZLOWSKI, P. et al. New applications and developments in the use of multiplex ligation-dependent probe amplification. Electrophoresis, v. 29, n. 23, p. 462736, 2008.

22. KREPISCHI-SANTOS, A. C.; VIANNA-MORGANTE, A. M. Disclosing the mechanisms of origin of de novo shortarm duplications of chromosome 9. Am J Med Genet $A$, v. 117A, n. 1, p. 41-6, 2003.

23. LEE, C. et al. Copy number variations and clinical cytogenetic diagnosis of constitutional disorders. Nat Genet, v. 39, n. 7 (Suppl), p. S48-54, 2007.

24. LINARDOPOULOU, E. V. et al. Human subtelomeres are hot spots of interchromosomal recombination and segmental duplication. Nature, v. 437, n. 7055, p. 94-100, 2005.

25. MILLER, D. T. et al. Consensus statement: chromosomal microarray is a first-tier clinical diagnostic test for individuals with developmental disabilities or congenital anomalies. Am J Hum Genet, v. 86, n. 5, p. 749-64, 2010.

26. MOOG, U. et al. Subtelomeric chromosome aberrations: still a lot to learn. Clin Genet, v. 68, n. 5, p. 397-407, 2005.

27. PIMENTEL, M. M. G. Síndrome do " $X$ " frágil: diagnóstico molecular. J Bras Patol, v. 35, n. 2, p. 94-98, 1999.

28. PINKEL, D. et al. High resolution analysis of DNA copy number variation using comparative genomic hybridization to microarrays. Nat Genet, v. 20, n. 2, p. 207-11, 1998.

29. RAUCH, A. et al. Molecular karyotyping using an SNP array for genomewide genotyping. J Med Genet, v. 41, n. 12, p. 916-22, 2004.

30. RIETHMAN, H. et al. Human subtelomere structure and variation. Chromosome Res, v. 13, n. 5, p. 505-15, 2005.

31. ROELEVELD, N. et al. The prevalence of mental retardation: a critical review of recent literature. Dev Med Child Neurol, v. 39, n. 2, p. 125-32, 1997.

32. SACCONE, S. et al. The highest gene concentrations in the human genome are in telomeric bands of metaphase chromosomes. Proc Natl Acad Sci USA, v. 89, n. 11, p. 4913-7, 1992.

33. SCHOUTEN, J. P. et al. Relative quantification of 40 nucleic acid sequences by multiplex ligation-dependent probe amplification. Nucleic Acids Res, v. 30, n. 12, p. e57, 2002.

34. SCHROCK, E. et al. Multicolor spectral karyotyping of human chromosomes. Science, v. 273, n. 5274 , p. 494-7, 1996.

35. SCHROCK, E. et al. Spectral karyotyping refines cytogenetic diagnostics of constitutional chromosomal abnormalities. Hum Genet, v. 101, n. 3, p. 255-62, 1997. 
36. SHAFFER, L. G. American College of Medical Genetics guideline on the cytogenetic evaluation of the individual with developmental delay or mental retardation. Genet Med, v. 7, n. 9, p. 650-4, 2005.

37. SHAFFER, L. G. Diagnosis of microdeletion syndromes by fluorescence in situ hybridization (FISH). Curr Protoc Hum Genet, chapter 8, unit 8.10, 2001.

38. SHAFFER, L. G. et al. Targeted genomic microarray analysis for identification of chromosome abnormalities in 1500 consecutive clinical cases. J Pediatr, v. 149, n. 1, p. 98-102, 2006.

39. SHAIKH, T. H. et al. High-resolution mapping and analysis of copy number variations in the human genome: a data resource for clinical and research applications. Genome Res, v. 19, n. 9, p. 1682-90, 2009.

40. SHAO, L. et al. Identification of chromosome abnormalities in subtelomeric regions by microarray analysis: a study of 5,380 cases. Am J Med Genet A, v. 146A, n. 17, p. 2242-51, 2008.

41. SHINAWI, M.; CHEUNG, S. W. The array CGH and its clinical applications. Drug Discov Today, v. 13, n. 17-18, p. 760-70, 2008.

42. SLATER, H. R. et al. High-resolution identification of chromosomal abnormalities using oligonucleotide arrays containing 116,204 SNPs. Am J Hum Genet, v. 77, n. 5, p. 709-26, 2005.
43. SOLINAS-TOLDO, S. et al. Matrix-based comparative genomic hybridization: biochips to screen for genomic imbalances. Genes Chromosomes Cancer, v. 20, n. 4, p. 399-407, 1997.

44. SPEICHER, M. R. et al. Karyotyping human chromosomes by combinatorial multi-fluor FISH. Nat Genet, v. 12 , n. 4, p. 368-75, 1996.

45. TONNIES, H. Modern molecular cytogenetic techniques in genetic diagnostics. Trends Mol Med, v. 8, n. 6, p. 246-50, 2002.

46. TRASK, B. J. Fluorescence in situ hybridization: applications in cytogenetics and gene mapping. Trends Genet, v. 7, n. 5, p. 149-54, 1991.

47. TRASK, B. J. Human cytogenetics: 46 chromosomes, 46 years and counting. Nat Rev Genet, v. 3, n. 10, p. 769-78, 2002.

48. TURNER, G. et al. Prevalence of fragile X syndrome. Am J Med Genet, v. 64, n. 1, p. 196-7, 1996.

49. VISSERS, L. E. et al. Array-based comparative genomic hybridization for the genomewide detection of submicroscopic chromosomal abnormalities. Am J Hum Genet, v. 73, n. 6, p. 1261-70, 2003.

50. XU, J.; CHEN, Z. Advances in molecular cytogenetics for the evaluation of mental retardation. Am J Med Genet C Semin Med Genet, v. 117C, n. 1, p. 15-24, 2003.

51. YUNIS, J. J. High resolution of human chromosomes. Science, v. 191, n. 4233, p. 1268-70, 1976. 